\title{
High-flux dialysis improves pulmonary ventilation function in uremic patients
}

\author{
HUI LIN ${ }^{1,2}$, YONG-GUI WU ${ }^{1}$, JIANG-HUAI ZHANG ${ }^{2}$ and MING KAN ${ }^{2}$ \\ ${ }^{1}$ Division of Nephrology, Department of Internal Medicine, The First Affiliated Hospital of Anhui Medical University, \\ Hefei, Anhui 230001; ${ }^{2}$ Division of Nephrology, Department of Internal Medicine, The Third Affiliated Hospital of \\ Anhui Medical University, Hefei, Anhui 230022, P.R. China
}

Received November 17, 2012; Accepted March 5, 2013

DOI: $10.3892 / \mathrm{mmr} .2013 .1373$

\begin{abstract}
Lung injury commonly accompanies uremia caused by renal failure. Uremia is typically treated using hemodialysis (dialysis) to restore electrolyte and fluid balance. A more recent, less commonly used method, high-flux dialysis, has not yet been investigated for its potential benefit to lung function. The aim of the present study was to determine whether high-flux dialysis affects pulmonary function. We assessed various pulmonary function parameters in patients with uremia before and after routine or high-flux dialysis. Pulmonary function was assessed via determination of the forced vital capacity (FVC), maximum breathing capacity (MBC), forced expiratory volume in 1 sec $\left(\mathrm{FEV}_{1}\right)$, peak expiratory flow (PEF), maximal midexpiratory flow (MMEF) curve, maximal expiratory flow in $25 \%$ vital capacity $\left(\mathrm{V}_{25}\right)$ and diffusion capacity of the lungs for carbon monoxide (DLco) in 42 patients with uremia and 24 healthy individuals. Patients with uremia were divided into two groups; the high-flux group (treated with high-flux dialysis; $n=21$ ) and the routine group (treated with conventional dialysis; $n=21$ ). Lung function was reassessed in the two groups after 3 months of dialysis. The two groups of patients with uremia exhibited reduced lung function parameters compared with healthy individuals (all $\mathrm{P}<0.05$ ), indicating the presence of impaired lung function secondary to uremia. Following dialysis, the $\mathrm{FEV}_{1}, \mathrm{PEF}$, MMEF and $\mathrm{V}_{25}$ values increased significantly compared with their respective baseline values prior to treatment for each group (ANOVA, $\mathrm{P}<0.05$ ). Furthermore, increases were more marked in patients treated with high-flux dialysis compared with those treated using routine dialysis $(\mathrm{P}<0.05)$. Thus, lung injury caused by uremia was shown to be improved following
\end{abstract}

Correspondence to: Dr Yong-Gui Wu, Division of Nephrology, Department of Internal Medicine, The First Affiliated Hospital of Anhui Medical University, 218 Jixi Road, Hefei, Anhui 230022, P.R. China

E-mail: wygui230022@126.com

Key words: high-flux hemodialysis, renal failure, pulmonary function dialysis, with high-flux dialysis offering a greater benefit than routine dialysis.

\section{Introduction}

Uremia, the build-up of nitrogenous waste following renal failure, commonly affects lung function (1). This condition leads to increased lung vascular permeability, inflammation and dysregulated salt and water channels, potentially through cytokine-mediated tissue crosstalk $(2,3)$. Several factors affect the extent of changes in pulmonary function in uremic individuals, including salt retention, an accumulation of toxic substances, anemia, malnutrition and a microinflammatory state (4-7). Notably, the management of these factors through blood purification (hemodialysis, dialysis), the correction of anemia, nutritional improvement and inflammation control improves lung function; dialysis is the preferred treatment for improving lung function in uremic patients (8).

Dialysis uses a porous membrane to transfer solutes and water to the blood, creating a gradient that aids the removal of urea and other waste products. The flow of particles across this membrane is partially determined by the size of the pores, since smaller pores result in lower flux and larger pores result in higher flux. High-flux dialysis is a more recent treatment that is increasingly being used in patient care due to the potential benefits on cardiac outcomes (9). However, to the best of our knowledge, no previous studies have investigated whether high-flux dialysis results in improved lung function in uremic patients.

In the present study, we assessed pulmonary function in 21 uremic individuals prior to and after high-flux dialysis treatment and compared their results with those of individuals treated with low-flux (routine) dialysis. Our results may have implications for the treatment of remote organ dysfunction in renal failure.

\section{Subjects and methods}

Study subjects. Forty-two uremic patients who were admitted to the Division of Nephrology, Department of Internal Medicine, The Third Affiliated Hospital of Anhui Medical University (Hefei, China) between January 2008 and August 2012 were enrolled in this study. None of the patients 
had been previously treated with dialysis or diagnosed with chronic bronchitis, bronchial asthma or other chronic respiratory diseases. The patients were equally divided into two treatment groups, the high-flux and routine groups, according to their order of admission; the first 21 patients were placed in the high-flux group and the second 21 in the routine group. Patients in the high-flux group (13 males and 8 females, aged $51.3 \pm 8.6$ years) underwent high-flux dialysis, while those in the routine group (14 males and 7 females, aged $50.8 \pm 7.9$ years) underwent routine dialysis. Additionally, 24 healthy individuals who underwent physical examination in the Third Affiliated Hospital of Anhui Medical University during the same period of time were included in this study to determine the differences in lung function between uremic patients and healthy individuals. The control group included 16 males and 8 females, aged $51.9 \pm 9.1$ years. Individuals in this group were free from chronic cardiopulmonary and renal disease, had normal cardiac and pulmonary function, as confirmed by physical examination, and had a normal chest X-ray and pulmonary function test. Age and gender were not significantly different among the groups. This study was approved by the Ethics Committee of the Third Affiliated Hospital of Anhui Medical University, and the written informed consent was obtained from the patients or their family members.

Treatment. Patients in the high-flux group were treated with an FX60 high-flux dialyzer and a high-flux helixone polysulfone membrane (membrane area, $1.4 \mathrm{~m}^{2}$; ultrafiltration coefficient, $46.0 \mathrm{ml} / \mathrm{h} / \mathrm{mmHg} / \mathrm{m}^{2}$ ), produced by Fresenius Medical Care (St. Wendel, Germany). Patients in the routine group were treated with an F7 dialyzer and a polysulfone membrane (membrane area, $1.6 \mathrm{~m}^{2}$; ultrafiltration coefficient, $16.0 \mathrm{ml} / \mathrm{h} / \mathrm{mmHg} / \mathrm{m}^{2}$ ). The $4008 \mathrm{~S}$ dialysis machine with an automatic volume control system (Fresenius), the ultrapure water bicarbonate dialysate (dialysate flow rate, $500 \mathrm{ml} / \mathrm{min}$; blood flow, 220-300 $\mathrm{ml} / \mathrm{min}$ ) and conventional heparin anticoagulation were used in the two groups. Dialysis was performed 3 times per week for $4 \mathrm{~h}$ each time, for 3 consecutive months. The dehydration amount depended on the condition of the patient. Conventional treatment with erythropoietin and active vitamin $\mathrm{D}$ and antihypertensive treatment were administered to patients in the two groups; the patients were monitored for 3 months. No treatments were administered to the healthy individuals in the control group.

Pulmonary function assessment. The pulmonary function of subjects in each of the three groups was assessed using the 6200 pulmonary function detector from Sensor Medics (Homestead, FL, USA). For the two treatment groups, lung function was assessed prior to and after 3 months of dialysis. Pulmonary function indicators included forced vital capacity (FVC), maximal breathing capacity (MBC), forced expiratory volume in $1 \mathrm{sec}\left(\mathrm{FEV}_{1}\right)$, peak expiratory flow (PEF), maximum middle expiratory flow (MMEF), peak expiratory flow at $25 \%$ vital capacity $\left(\mathrm{V}_{25}\right)$ and the diffusion capacity of the lungs for carbon monoxide (DLco). Measured values are expressed as percentages $(\%)$ of normal expected values; pulmonary function was determined by the first author of this study.

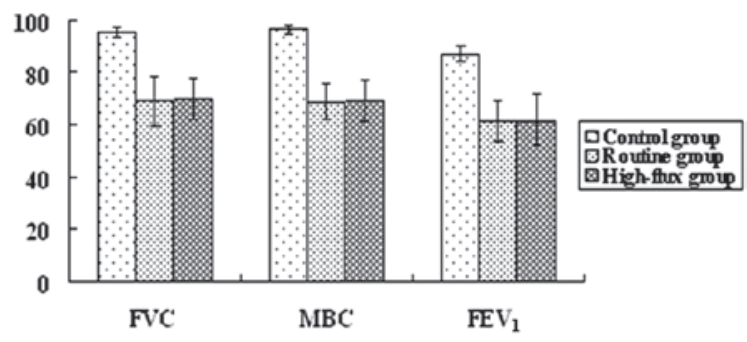

Figure 1. Baseline pulmonary function in healthy individuals (control group) compared with uremic patients (the routine and high-flux groups). $\mathrm{FVC}$, forced vital capacity; MBC, maximal breathing capacity; $\mathrm{FEV}_{1}$, forced expiratory volume in $1 \mathrm{sec}$.

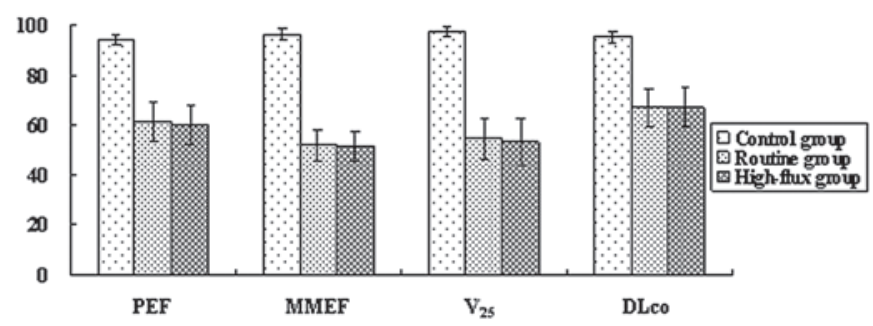

Figure 2. Peak expiratory flow (PEF), maximum midexpiratory flow (MMEF), peak expiratory flow at $25 \%$ vital capacity $\left(\mathrm{V}_{25}\right)$ and diffusion capacity of the lungs for carbon monoxide (DLco) in all three groups.

Statistical analysis. SPSS 17.0 was used for statistical analysis. Measurement data are expressed as the mean \pm standard deviation (SD). The $\chi^{2}$ test and one-way ANOVA were used to compare gender, age and pulmonary function prior to dialysis among the three groups. Variance analysis using repeated data measurements was used to compare pulmonary function prior to and after dialysis in the high-flux and routine groups, separately, and to compare pulmonary function between the two groups. Tests were two-sided with $\alpha=0.05$ and $\mathrm{P}<0.05$ was considered to indicate a statistically significant difference.

\section{Results}

Baseline pulmonary function differs between uremic patients and healthy individuals. Prior to uremia treatment, pulmonary function was assessed to determine the baseline lung function of patients in the two treatment groups compared with that of healthy individuals. For each parameter (FVC, MBC, FEV PEF, MMEF, $\mathrm{V}_{25}$ and DLco), uremic patients in the high-flux and routine groups exhibited significantly lower values compared with the healthy population $(\mathrm{P}<0.05$; Figs. 1 and 2$)$.

High-flux dialysis improves pulmonary function. The pulmonary function of uremic patients was reassessed following 3 months of dialysis treatment. After either high-flux or routine dialysis, several parameters of pulmonary function were significantly increased compared with their respective baseline values $\left(\mathrm{FEV}_{1}\right.$, PEF, MMEF, $\mathrm{V}_{25}$ in the high-flux and routine groups; all $\mathrm{P}<0.05$; Table I). Additionally, these parameters were significantly improved in uremic patients treated with high-flux dialysis compared with those who underwent routine dialysis (all $\mathrm{P}<0.05$ ). No changes were observed in FVC, MBC or DLco. 
Table I. Comparison of pulmonary function between the high-flux and routine dialysis groups at baseline and post-treatment.

\begin{tabular}{|c|c|c|c|c|c|c|c|}
\hline Dialysis type & FVC & $\mathrm{MBC}$ & $\mathrm{FEV}_{1}$ & PEF & MMEF & $\mathrm{V}_{25}$ & DLco \\
\hline \multicolumn{8}{|l|}{ Routine } \\
\hline Baseline $(\% ; \mathrm{n}=21)$ & $69.1 \pm 9.7$ & $68.9 \pm 6.5$ & $61.2 \pm 8.0$ & $61.4 \pm 7.7$ & $52.0 \pm 6.2$ & $54.5 \pm 8.1$ & $66.9 \pm 7.4$ \\
\hline Post-treatment $(\% ; \mathrm{n}=21)$ & $73.0 \pm 4.7$ & $72.3 \pm 7.3$ & $67.3 \pm 9.7$ & $78.9 \pm 6.1$ & $71.5 \pm 6.4$ & $74.3 \pm 7.2$ & $68.9 \pm 7.0$ \\
\hline F-value & 2.572 & 3.524 & 6.157 & 101.878 & 119.585 & 90.945 & 1.331 \\
\hline P-value & 0.124 & 0.075 & 0.022 & 0.001 & 0.001 & 0.001 & 0.262 \\
\hline \multicolumn{8}{|l|}{ High-flux } \\
\hline Baseline $(\% ; \mathrm{n}=21)$ & $69.7 \pm 7.7$ & $69.0 \pm 7.9$ & $61.9 \pm 9.7$ & $60.1 \pm 7.8$ & $51.5 \pm 6.0$ & $53.1 \pm 9.3$ & $67.0 \pm 7.7$ \\
\hline Post-treatment $(\% ; \mathrm{n}=21)$ & $73.9 \pm 7.4$ & $74.1 \pm 7.7$ & $76.1 \pm 9.1$ & $87.7 \pm 6.5$ & $78.3 \pm 6.1$ & $83.8 \pm 7.6$ & $70.1 \pm 9.4$ \\
\hline F-value & 2.879 & 3.714 & 22.282 & 143.016 & 258.185 & 155.198 & 1.669 \\
\hline P-value & 0.105 & 0.068 & 0.001 & 0.001 & 0.001 & 0.001 & 0.211 \\
\hline F-value & 0.220 & 0.335 & 5.398 & 5.457 & 4.565 & 4.543 & 0.106 \\
\hline $\mathrm{P}$-value & 0.642 & 0.566 & 0.025 & 0.025 & 0.039 & 0.039 & 0.746 \\
\hline
\end{tabular}

Measurement results are expressed as the mean \pm standard deviation (SD). FVC, forced vital capacity; MBC, maximal breathing capacity; $\mathrm{FEV}_{1}$, forced expiratory volume in $1 \mathrm{sec}$; PEF, peak expiratory flow; MMEF, maximum midexpiratory flow; $\mathrm{V}_{25}$, peak expiratory flow at $25 \%$ vital capacity; DLco, diffusion capacity of the lungs for carbon monoxide.

\section{Discussion}

The incidence of lung injury in uremic patients has been reported to be $>46.6 \%$ (10). Previous studies on the effects of uremia on pulmonary function have demonstrated decreased pulmonary diffusion, while results for pulmonary ventilation function are inconsistent $(11,12)$. In the present study, uremic patients exhibited significantly lower pulmonary function indices compared with the healthy population prior to dialysis; the pulmonary ventilation (FVC, MBC), large- and medium-sized airway function $\left(\mathrm{FEV}_{1}, \mathrm{PEF}\right)$ and small airway function indices (MMEF, $\mathrm{V}_{25}$ ) were all indicative of impaired lung function. Additionally, the pulmonary diffusion capacity index (DLco) was significantly impaired. These results indicate that uremic patients experience pulmonary ventilation and diffusion dysfunction and airway obstruction, particularly in small airways. These results may be explained by the accumulation of water and toxic substances in the body under uremic conditions, which increases the permeability of alveolar capillaries. This allows the superabundant liquid to transfer from the pulmonary blood vessels to the pulmonary interstitium, causing interstitial edema, the increased osmotic pressure of alveolar capillaries, the hydrostatic pressure of pulmonary vessels and the ventilation dysfunction of large and small airways, which manifests as restrictive ventilation dysfunction. Diffusion function has also been shown to decrease due to pulmonary interstitial and alveolar edema $(11,13)$.

To assess the effects of dialysis and, more specifically, to compare the effects of high-flux and routine dialysis on uremia-induced lung injury, the lung function of uremic patients was reassessed following high-flux or routine dialysis. Of the 7 lung function parameters assessed, four $\left(\mathrm{FEV}_{1}\right.$, $\mathrm{PEF}, \mathrm{MMEF}$ and $\mathrm{V}_{25}$ ) were significantly increased in the two treatment groups compared with their respective baseline (pre-treatment) values. However, FVC, MBC and DLco were not altered. These results indicate that dialysis expels excessive toxins and water, restores electrolyte and acid-base balance, reduces the permeability of pulmonary capillaries and relieves pulmonary edema, thereby improving pulmonary ventilation in uremic patients (14).

High-flux dialysis has an extremely high diffusion performance and hydraulic permeability, and is able to eliminate $\beta 2$ microglobulin from the body of uremic patients, reduce blood phosphorus, blood fat and parathyroid hormone and relieve inflammation, oxidative stress and vascular endothelial changes. This approach is superior to conventional dialysis in eliminating urea nitrogen, creatinine and other small molecules (15). In the present study, high-flux dialysis was also superior in the extent of improvement of pulmonary function in uremic patients. This result provides additional evidence to support the implementation of high-flux dialysis in clinics, as it provides a greater benefit in treating uremia-induced lung injury. Notably, dialysis is a replacement therapy that is unable to be performed in a persistent state, and hemodynamic and biological compatibility changes may occur during the course of this type of treatment. These factors may also affect the pulmonary function of patients; thus, it is difficult to fully restore pulmonary function through dialysis.

In conclusion, high-flux dialysis improves the function of large and small airways and pulmonary ventilation, and offers a more marked improvement compared with routine dialysis. Therefore, high-flux dialysis should be considered for clinical use as it may significantly benefit patients by offering an improved quality of life.

\section{References}

1. Turcios NL: Pulmonary complications of renal disorders. Paediatr Respir Rev 13: 44-49, 2012.

2. Scheel PJ, Liu M and Rabb H: Uremic lung: new insights into a forgotten condition. Kidney Int 74: 849-851, 2008.

3. Doi K, Ishizu T, Fujita T and Noiri E: Lung injury following acute kidney injury: kidney-lung crosstalk. Clin Exp Nephrol 15: 464-470, 2011. 
4. Jean G: How to manage mineral metabolism disorders in renal failure. Presse Med 40: 1043-1052, 2011 (In French).

5. Artunc F and Risler T: Serum erythropoietin concentrations and responses to anaemia in patients with or without chronic kidney disease. Nephrol Dial Transplant 22: 2900-2908, 2007.

6. Nascimento MM, Qureshi AR, Stenvinkel P, et al: Malnutrition and inflammation are associated with impaired pulmonary function in patients with chronic kidney disease. Nephrol Dial Transplant 19: 1823-1828, 2004

7. Razeghi E, Pazoki M, Ahmadi F, et al: Relation between pulmonary function and inflammatory biomarkers in hemodialysis patients. Ren Fail 34: 24-27, 2012.

8. Karacan O, Tutal E, Uyar M, et al: Pulmonary function in uremic patients on long-term hemodialysis. Ren Fail 26: 273-278, 2004.

9. Eknoyan G, Beck GJ, Cheung AK, et al: Effect of dialysis dose and membrane flux in maintenance hemodialysis. $\mathrm{N}$ Engl J Med 347: 2010-2019, 2002.
10. Wanner C and Ketteler M: Chronic renal failure. Dtsch Med Wochenschr 136: 1591-1593, 2011 (In German).

11. Kovelis D, Pitta F, Probst VS, et al: Pulmonary function and respiratory muscle strength in chronic renal failure patients on hemodialysis. J Bras Pneumol 34: 907-912, 2008.

12. Bush A and Gabriel R: Pulmonary function in chronic renal failure: effects of dialysis and transplantation. Thorax 46: 424-428, 1991

13. Dujić Z, Eterović D and Tocilj J: Pulmonary function in chronic renal failure: effects of dialysis and transplantation. Thorax 47: 763, 1992.

14. Prezant DJ: Effect of uremia and its treatment on pulmonary function. Lung 168: 1-14, 1990.

15. Palmer SC, Rabindranath KS, Craig JC, et al: High-flux versus low-flux membranes for end-stage kidney disease. Cochrane Database Syst Rev 9: CD005016, 2012. 